\title{
- Profitability in the car industry: new measures for estimating targets and target directions
}

\author{
Hans van der Heijden, Wolfgang Garn \\ The Surrey Business School, University of Surrey, Guildford, Surrey, GU2 7XH, United Kingdom
}

\section{Abstract}

In this paper we study the profitability of car manufacturers in relation to industry-wide profitability targets such as industry averages. Specifically we are interested in whether firms adjust their profitability in the direction of these targets, whether it is possible to detect any such change, and, if so, what the precise nature is of these changes.

This paper introduces several novel methods to assess the trajectory of profitability over time. In doing so we make two contributions to the current body of knowledge regarding the dynamics of profitability. First, we develop a method to identify multiple profitability targets. We define these targets in addition to the commonly used industry average target. Second, we develop new methods to express movements in the profitability space from $t$ to $t+j$, and define a notion of agreement between one movement and another.

We use empirical data from the car industry to study the extent to which actual movements are in alignment with these targets. Here we calculate the three targets that we have previously identified, and contrast them with the actual profitability movements using our new agreement measure. We find that firms tend to move more towards to the new targets we have identified than to the common industry average.

Keywords: Forecasting, DuPont ratios, Multivariate statistics, Kernel Density Estimation, Directional Statistics, Profitability Targets

\section{Introduction}

Understanding the drivers of business profitability has been a longstanding domain of interest for operational researchers (see e.g., Wu et al. (2010); Tecles and Tabak (2010); So and Thomas 
(2011) for recent examples). In this paper we study the profitability of individual firms in relation to industry-wide profitability targets such as industry averages. Specifically we are interested in whether firms adjust their profitability in the direction of these targets, whether it is possible to detect any such change, and, if so, what the precise nature is of these changes.

Our study of profitability limits itself to the two DuPont profitability ratios: profit margin and asset turnover. The DuPont profitability ratios are disaggregated components of Return on Assets (ROA). The ratios are well-documented in textbooks, and serve as the basic building blocks for profitability. Profit margin is defined as net income divided by sales. Asset turnover is defined as sales divided by total assets. In this paper we follow the approach advanced by Penman (2010) to focus on Return on Net Operating Assets (RNOA), in order to neutralize the ways in which firms use financial leverage to increase profit. Financial leverage influences overall profitability by incorporating profits from financial assets, and because these profits can vary widely between companies in the same industry, it is difficult to compare operating profitability using ROA data. In line with this approach we study the disaggregated, multiplicative components of RNOA: Operating Profit Margin (OPM) and Net Operating Asset Turnover (ATO). For ease of reference, the study will often simply refer to these components as profit margin and asset turnover.

It is useful to visualize these two profitability drivers in a two-dimensional plane, with ATO on the $\mathrm{X}$-axis and OPM on the $\mathrm{Y}$-axis. The various RNOA $c$ levels (where $\mathrm{OPM} \times \mathrm{ATO}=c$ ) can then be depicted in the form of iso-curves. Soliman (2004) and Penman (2010) provide illustrations of such plots. The two-dimensional plane is an instance of a more general $n$-dimensional profitability space, in this case with $n=2$. We are interested in describing how firms move year on year through this space (i.e., from one ATO-OPM point to another), and whether this movement is influenced by certain target points in the space.

This paper introduces several novel methods to assess the trajectory of profitability over time. In doing so we make two contributions to the current body of knowledge regarding the dynamics of profitability.

First, we develop a method to identify multiple profitability targets. We define these targets in addition to the commonly used industry average target. The derivation of our new targets is based 
on linear diffusion of kernel density estimation (KDE). KDE has the advantage that it does not estimate one global maximum (i.e., one "peak") but instead allows for the possibility of multiple local maxima. These local maxima manifest themselves as multiple, local "hills" in the profitability space. Using the KDE estimator we arrive at two new types of targets in addition to the industry average target.

Second, we develop new methods to express movements in the profitability space from $t$ to $t+j$, and define a notion of agreement between one movement and another. The method is based on a comparison of the angles of movement, and a mapping of the difference in angles onto a linear $[-1,1]$ domain. The result is an agreement measure which enables us to express one profitability movement as a percentage of agreement with another movement.

The target level is often taken to be the profitability mean of the industry in which the firm operates. Previous research in this area has looked at whether the ratios are mean-reverting over time (see for example Freeman et al. (1982)). Lev (1969) provides the first empirical evidence that firms do indeed adjust their ratios to such target levels. Lev also discusses the difficulty of adjustment (in the sense that some ratios are easier to manage than others) and the cost of not adjusting, for example, if banks insist on target levels and raise loan interest if the firm does not meet these levels.

Other than creditor pressure, theoretical reasons for firms moving their profitability towards target levels can be found in the competitive forces framework as outlined by Porter $(1980,1985)$. If the profitability of one firm is much higher than its peers, competing firms will attempt to imitate the distinctive resources available to the superior firm, or will move into the arena where the superior company enjoyed above-average profits. If the profitability of a firm drops below those of its peers, the firm will be much less profitable than the competition and it will face the risk of failure or takeover.

Fama and French (2000) use a version of the partial adjustment model which allows crosssectional data to be combined with time series data, leading to a larger sample to draw conclusions from. They find strong evidence that profitability (return on assets) is indeed mean-reverting. They also show that firms with much higher profitability tend to revert faster. Soliman (2004), using a 
comprehensive empirical study, tests whether OPM and ATO indeed revert to their industry means rather than to the general economy-wide levels. The study finds increased predictive ability of RNOA when taking into account industry adjustments for OPM and ATO. Soliman concludes that it is worthwhile to study OPM and ATO at the industry level; this is precisely the approach adopted in this paper.

Related research has focused on the incremental benefit of looking at the disaggregated profitability ratios OPM and ATO and their informativeness for predicting future earnings. Fairfield and Yohn (2001) study changes in profitability and look at the incremental benefit of ATO and OPM specifically. They find that disaggregating the change in return on assets into the change in ATO and the change in OPM helps to better predict future profitability. Soliman (2008) similarly finds the profitability measures to be informative for stock market prices.

We use empirical data from the car industry to study the extent to which actual movements are in alignment with these targets. The automobile sector has been subject of research on financial performance before (see e.g., Saranga (2009) for an example in the component manufacturing industry). We focus on the 21 US, Japanese and German car manufacturers with a global presence. For each firm we calculate the three targets that we have previously identified, and contrast them with the actual profitability movements using our new agreement measure. We find that firms tend to move more towards the new targets we have identified than to the common industry average.

The remainder of the paper is as follows. We first present the new methods for profitability targets, profitability movements and directional agreements. We then document our sample and present the results of the calculations. Finally we present conclusions and directions for future research.

\section{Method Development}

\subsection{Movements and directional agreement}

The first step in the development of our method is the standardization of the two profitability ratios. If left unstandardized, unit changes have differential effects on the two profitability ratios. For instance if the ATO range is 10 and the OPM range is 0.1 then without scaling (standardizing) 
them ATO has a 100 times larger impact, any OPM change would be negligible. It is therefore important to establish the relative weighting of ATO and OPM. Our assumption here is that they have equal weight.

We standardize every ratio by their yearly min-max range, such that unit changes carry the same impact for every ratio. The scaling is accomplished by dividing the ATO and OPM distances by their yearly ranges. The ATO range is $\max \left\{A T O_{t}\right\}-\min \left\{A T O_{t}\right\}$ and analogously for the OPM range. Here, these ranges will be called scaling factors and denoted by $s:=\left(s_{A T O}, s_{O P M}\right)$.

The actual profitability movement of a company $c$ is its change of ATO and OPM from year $t$ to year $t+j$, where $j$ is the number of years forward.

The vector

$$
\begin{aligned}
\overrightarrow{r_{c t}} & :=\left(r_{c, t+j}-r_{c, t}\right) \div s \\
& =\left(\mathrm{OPM}_{c, t+j}-\mathrm{OPM}_{c, t}, \mathrm{ATO}_{c, t+j}-\mathrm{ATO}_{c, t}\right) \div s
\end{aligned}
$$

will be defined as the actual direction of profitability: from one position in year $t$ to the following year $t+j$. The $\div$ operator indicates the element-by-element division by the scaling factor $s$. Given a current profitability position and a target position $\tau$ we can similarly define a target direction $\overrightarrow{\tau_{c t}}$.

We determine the level of agreement of the actual direction and target direction by considering the angles of the directions. Let $\varphi_{a}$ be the angle of the actual direction $\overrightarrow{r_{c t}}$ and $\varphi_{\tau}$ be the angle of the target direction $\overrightarrow{\tau_{c t}}$. The absolute difference between these angles is the difference angle $\Delta_{\varphi}$ :

$$
\Delta_{\varphi}=\left|\varphi_{a}-\varphi_{\tau}\right|
$$

To aid in our understanding of these difference angles, it is convenient to map them to an interval $[-1,1]$, where:

$$
\Psi \in \begin{cases}(0,1], & \text { directional agreement; } \\ 0, & \text { orthogonal; } \\ {[-1,0),} & \text { directional disagreement. }\end{cases}
$$




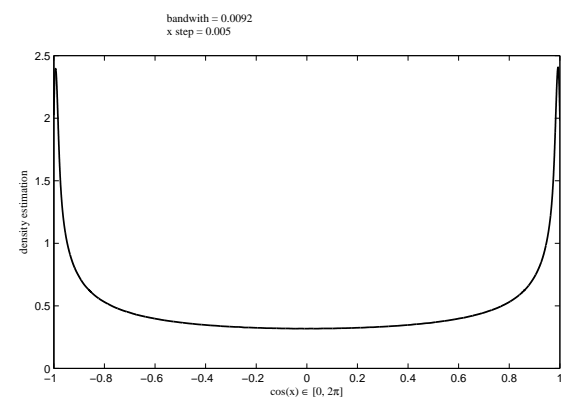

Figure 1: Alternative directional agreement measure based on the cosine function.

That means if actual and target direction are the same, then $\Psi=1$, and there is $100 \%$ agreement. In case they are orthogonal, then $\Psi=0$. If they point into opposite directions then the directional agreement is $\Psi=-1$, and the movements are in $100 \%$ disagreement.

This mapping is achieved by introducing the linear $\Psi$ function, which we will call directional agreement:

$$
\Psi:= \begin{cases}1-\frac{2 \Delta_{\varphi}}{\pi}, & \Delta_{\varphi} \leq \pi \\ \frac{2 \Delta_{\varphi}}{\pi}-3 & \Delta_{\varphi}>\pi\end{cases}
$$

There are alternative mappings. For instance one could use the cosine function. However, the density shape of the cosine measure is biased towards one and minus one (see figure 1). The linear mapping is chosen specifically to neutralize such biases.

Figure 2 presents a visual overview of these concepts. The Figure depicts one company (BMW), which moves in the profitability space from time $t=2010$ to time $t=2011$. The actual movement is in red. The target (here the industrial average) is in green, and the dotted green line represents a target movement. The blue lines represent orthogonal and opposite directions.

\subsection{Estimating profitability target}

Probability density estimations fall into two main classes: parametric and non-parametric estimation. Parametric estimations take a certain parametric distribution (e.g. normal, logistic, etc.) and determine parameters such that the error to the original sample data is minimized. Usually parametric distribution estimations have a unique maximum without local peaks. Ratios such as profit margins and asset turnovers do in general not follow the normal distribution and often posses local peaks. Hence,we use a non-parametric (i.e., distribution free) method. In this work we focus 


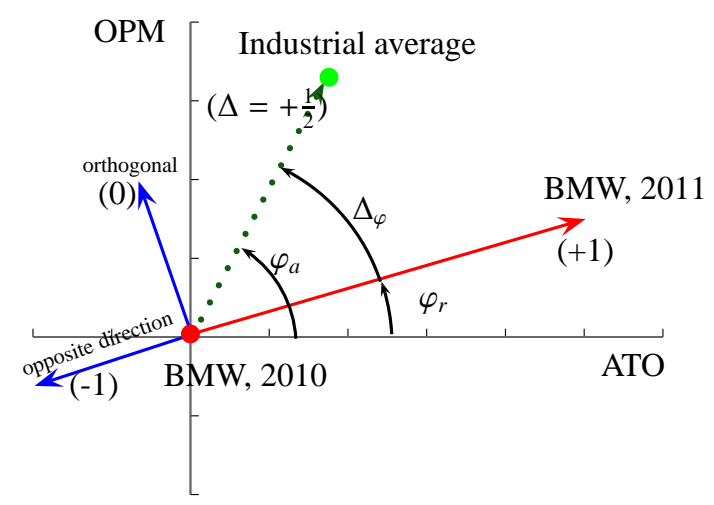

Figure 2: Development directions and angles.

on Kernel density estimators (non-parametric method) to achieve this goal.

Histograms are among the most traditional of discrete density estimations. Constructing histograms gives rise to the problem of defining bins having the "appropriate" width and location. In order to blur these hard bin boundaries, we can step a distance $s$ from the minimum to the maximum observation. At each step $x_{k}=x_{k-1}+s$ we count the number of observations in a surrounding interval $\left[x_{k}-w, x_{k}+w\right)$. That means there is an overlap, which means we count the observations twice, if $s=w$. This is called the naive classifier after normalization (division by $2 n w$ ):

$$
y_{k}=\frac{1}{2 n w} \sum_{k=1}^{n}\left[\left|x_{k}-\tilde{y}_{i}\right|<w\right],
$$

where $n$ is the number of observations, $2 w$ is the interval width (also known as window width, bandwidth or smoothing parameter) and $\tilde{y}$ is the vector of observations. The square brackets denote the Iverson convention, i.e. if the expression within the square brackets is true the returned value is one otherwise zero. Figure 3 shows a histogram and naive density estimates for OPM of car manufacturers for the years 1991 until 2010. The difference between the two naive estimates is that one has the window width $w$ set equal to the step size $s$.

An obvious weakness of the naive density estimate is its discontinuity at the interval boundaries, even when we choose half open intervals. However, if there are no observations at the interval 


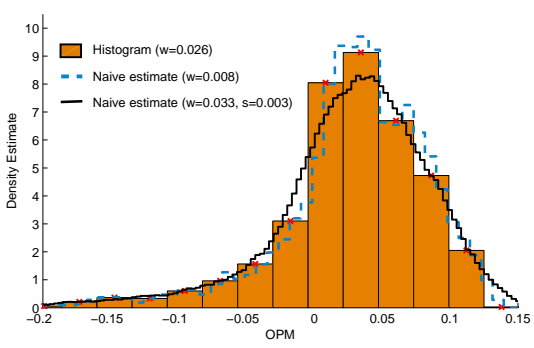

(a)

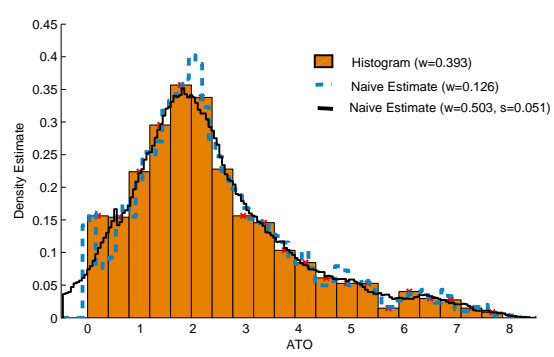

(b)

Figure 3: Histogram and naive density estimates for (a) OPM and (b) ATO of car manufacturers.

boundaries then probability density must equal to one:

$$
\sum_{k=1}^{n} s y_{k} \stackrel{!}{=} 1
$$

\footnotetext{
${ }^{1}$ In a more general notation equation (7) is written as $\sum_{-\infty}^{\infty} s y_{x} \delta x \stackrel{!}{=} 1$.
} 


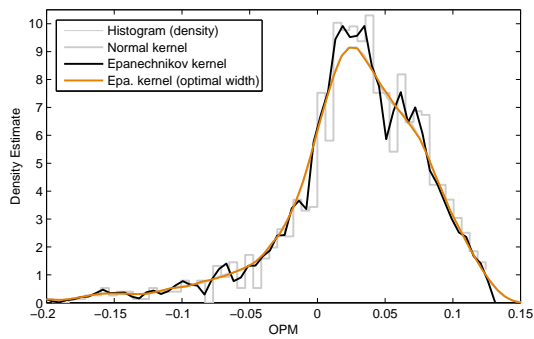

(a)

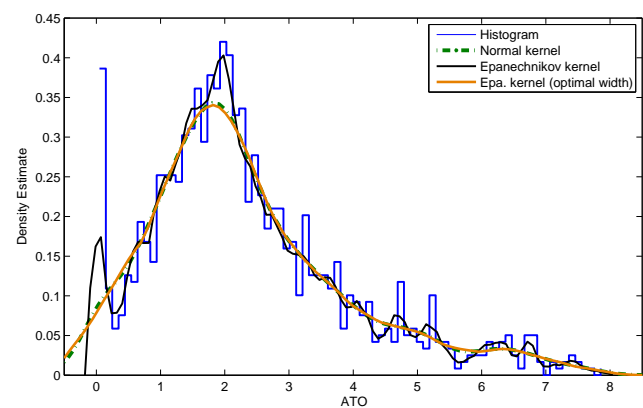

(b)

Figure 4: Typical density estimates for (a) OPM and (b) ATO of car manufacturers.

For this study we use a multivariate adaptive kernel density estimator, which is based on the linear diffusion process. This estimator was originally introduced by Botev et al. (2010). The bandwidth was determined by a plug-in selection method that is not based on normal reference rules. So the bandwidth selection is free of distribution assumptions. Going away from the mostly used Gaussian kernel density estimator also improves the local adaptivity. That means peaks are better reflected. Furthermore the linear diffusion KDE overcomes boundary issues most other KDE suffer from. The Appendix provides more specific details about this estimator.

\subsection{Movements towards targets}

Our objective is to compare movements from one (ATO,OPM) point to another. Therefore, we define four targets of interest. One of them is the actual (historic) movement of the profitability ratios. We will call $r_{c t}:=\left(A T O_{c t}, O P M_{c t}\right)$ the position of a company $c$ at year $t$. We are interested in getting to the following four targets, with the last two targets being new and developed using the Kernel Density Estimation:

- next position of actual financial ratios $r_{c t+p}$

- industrial average $\bar{a}_{t}$,

- density center $d_{t}$,

- maximum neighbor $m_{c t}$. 
The next position of an actual movement $r_{c t+p}$ is the ATO and OPM for the year $t+j$, where $j$ is the number of years in the future (e.g. one, three or five).

The industry average is determined each year by:

$$
\bar{a}_{t}:=\frac{1}{n} \sum_{c=1}^{n} r_{c t},
$$

where $n$ is the number of companies in year $t$.

The direction to the industry average is:

$$
\overrightarrow{a_{c t}}:=\left(a_{t}-r_{c t}\right) \div s,
$$

where $s$ was defined in section 2.1.

The density center $d_{t}$ is determined using the linear diffusion Kernel density estimation (KDE):

$$
d_{t}:=\operatorname{argmax}\left\{K D E\left(r_{t}, k\right)\right\},
$$

where $r_{t}:=\left(r_{1 t}, \ldots, r_{n t}\right)$. The KDE returns a heatmap $H$, which is a $\mathbb{R}^{k \times k}$ matrix with $k$ being a power two value (e.g. 256). Additionally we will know the $x$ and $y$ positions of the heat map values, which are $h_{x}$ and $h_{y}$. That means equation (11) returns as argument the position of the maximum probability density value. Note that uniqueness is not ensured.

Analogously we define the direction to the density center:

$$
\overrightarrow{d_{c t}}:=\left(d_{t}-r_{c t}\right) \div s
$$

and to the maximum neighbor:

$$
\overrightarrow{m_{c t}}:=\left(m_{c t}-r_{c t}\right) \div s
$$

The maximum neighbor $m_{c t}$ is the value which assumes a maximum in a neighborhood of $r_{c t}$ :

$$
m_{c t}:=\operatorname{argmax}\left\{\mathcal{N}\left(r_{c t}, K D E\left(r_{t}, k\right)\right)\right\}
$$


where $\mathcal{N}$ is an algorithm that returns a number of neighbors of $r_{c t}$. First all distances of $r_{c t}$ to $h_{x}$ and $h_{y}$ are determined. Then a selection (neighborhood) of the closest points with their probability density estimate is returned.

The kernel density estimations were implemented in Matlab using the ksdensity, kde (univariate) and kde2d (bivariate) functions. These functions implement algorithms published in Bowman and Azzalini (1997) and Botev et al. (2010) respectively.

\section{Data}

Because the study focuses on the development and profitability within industry level, we choose one specific industry to illustrate our techniques over a relatively long period of time. The industry we study is the car manufacturing industry, and the time horizon of the study is 16 years, from 1995 to 2010.

We studied the profitability measures of 31 global car manufacturers over the course of 16 years: 14 car manufacturers were from Japan, 9 from the United States, and 8 from Germany. The Worldscope database (ThomsonReuters) provides the underlying financial statement data for the analysis.

The calculation of operating profit margin and asset turnover closely follows Soliman (2008). Operating profit margin (OPM) is operating income (Worldscope datatype 1250) divided by net revenues (datatype 1001). Asset turnover (ATO) is net revenues divided by average net operating assets (NOA). NOA is operating assets minus operating liabilities. Operating assets are total assets (datatype 2999) less cash and short term investments (datatype 2001). Operating liabilities are total assets less long-term debt (3251), short-term debt (3051), common equity (3501), preferred stock (3451), and minority interest (3426). Average $N O A$ is $0.5\left(N O A_{t}+N O A_{t-1}\right)$ where $N O A_{t-1}$ is available, and $N O A_{t}$ where $N O A_{t-1}$ is not available.

Several invalid data points are encountered with the data: missing values, infinite values, zero values and outliers. To determine the yearly KDE, our approach was to remove these invalid data points on a year-by-year basis. For determining directionality it is necessary to ensure the validity of the data for both the selected year $t$ and the target year $t+j$ jointly. Furthermore, it is necessary 
that these issues are resolved for ATO and OPM concurrently for each company. The removal of nonexistent and infinite values is trivial. In theory zero values could happen, however in practice they should not. Thus we remove values that are exactly zero. "Outliers" are by far the most critical issue. ATO ratios were accepted if they were within the range -1 and 9. OPM ratios were required to be in the interval $(-0.4,0.2)$. Before discussing alternatives let us have a look at the effect of this constraints. Before processing the data there are 31 companies over 16 years, i.e. 496 possible values. 96 OPM, 111 ATO and 112 OPM \& ATO are infinite or nonexistent. 2 OPM, 26 ATO and 28 OPM \& ATO are zero. Boolean combination leaves us with 359 values. The range constraint removes another 41 values. So from the original data set $64 \%$ remain. This means that the number of companies per year varies between 17 and 23 within the 16 years.

Alternatives to handling outliers were considered: using outliers, confidence intervals (e.g. mean \pm 2 standard deviations), quantiles in [5\%,95\%] and a distance measure. The distance measure determines the average distance between values and removes those data points, which are beyond the average distance plus the standard deviation. However, the outlier handling with the range constraints preserves more data than its alternatives.

Thus, ATO and OPM are in distinct ranges with typical OPM ratios for car manufacturers in $[-0.4,0.2]$, whilst ATO ratios are within the range [-1,9]. This re-emphasizes the importance of scaling as a first step to the analysis: if no scaling is applied a change in the ATO ratio would have a roughly 17 times higher impact than a change in the OPM ratio.

\section{Results}

\subsection{Profitability Target Estimator}

Figure 5 (a) presents the bivariate kernel density estimation for the sample for the year 2008 . The figure is a three-dimensional representation of the OPM and ATO probability density estimation.

The figure is best interpreted as a smoothed-out bivariate histogram. We can see that there are local maxima (hills and peaks) with the highest maximum representing the center of the density function. Several firms (shown as red dots) cluster around a separate local maximum. 


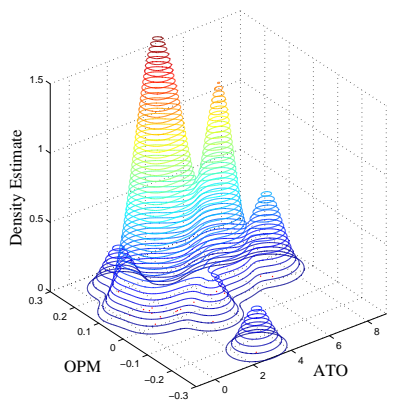

(a)

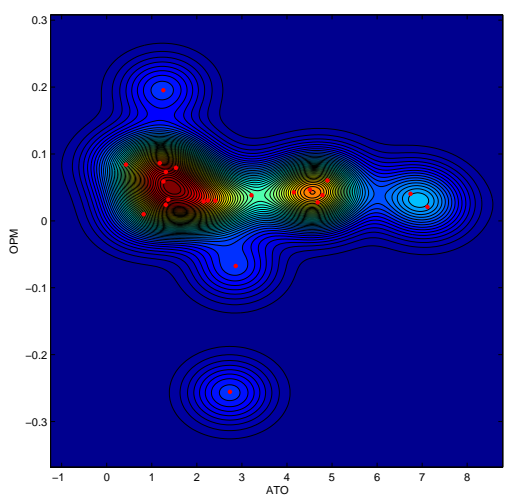

(b)

Figure 5: Probability density estimate of ATO and OPM for the year 2008.

Although the three-dimensional picture is useful to examine the local maxima in terms of height, it may also be misleading. This is because 3D pictures may distort, and in addition some local maxima may "hide" behind larger maxima. Figure 5 (b) presents a different visualization of the same density estimation. We will use this visualization in the remainder of the paper because its interpretation is not handicapped by possible 3D distortions. In addition, it matches the traditional depiction of ATO and OPM as discussed previously.

Initially we will analyze and describe in some detail the directional agreement of companies to their targets in the year 2008. The procedure used for that year will be applied for the whole period of 16 years. During this period (1995 until 2009) the industry average varies more than the density centers (see figure 6). The movements for the density centers are roughly $10 \%$ of the displayed range. The industry averages have particular large deflections in the years 1995, 1996, 2007 and 2009. This means taking industry average and density center as a target requires a renewed calculation on a year-on-year basis.

\subsection{Movements towards targets}

In order to evaluate the directional agreement we have to determine probability density estimates and the directions.

The vector map in figure 7 demonstrates the directions of the companies to the industrial average, density center and maximum neighbors in 2008. In particular the actual development of 


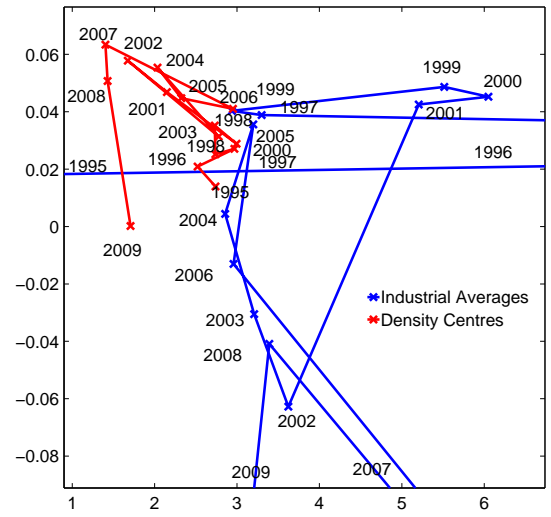

Figure 6: Moving targets: Industry averages and density centers for the period 1999-2009.

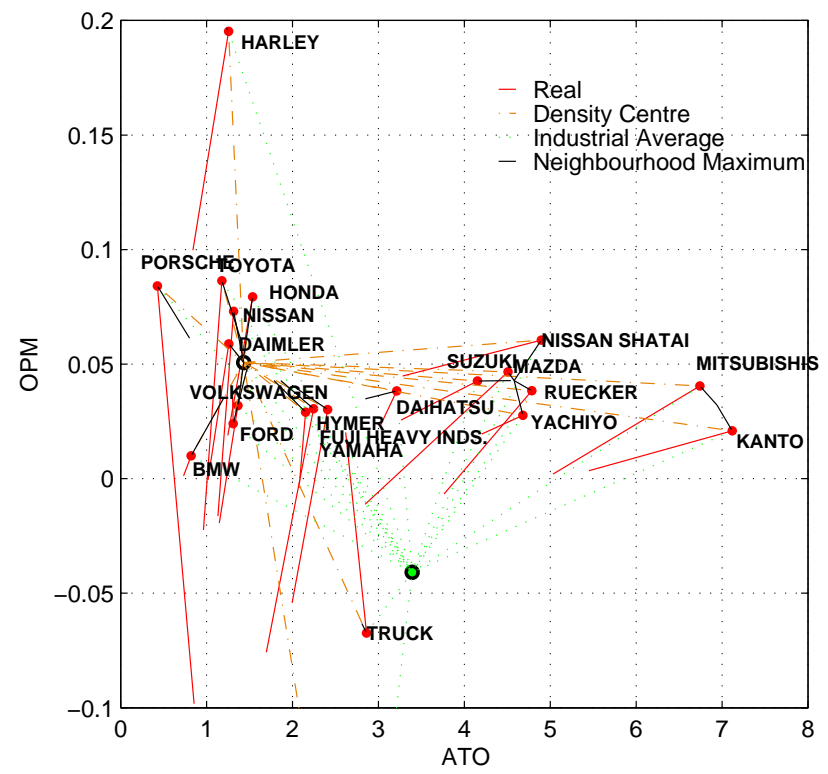

Figure 7: Directions of German, USA and Japanese car manufacturers in 2008. 
Table 1: Directional agreement, three targets vs actual movement 2008.

\begin{tabular}{rr|rrr}
\hline Directional & Statistic & density & neighbor & ind. avg. \\
\hline Agreement & Mean & 0.67 & 0.556 & 0.59 \\
& Std & 0.21 & 0.3 & 0.21 \\
& Median & 0.706 & 0.62 & 0.559 \\
\hline Disagreement & Mean & -0.533 & -0.483 & -0.444 \\
& Std & 0.38 & 0.32 & 0 \\
& Median & -0.359 & -0.428 & -0.444 \\
\hline
\end{tabular}

a company to the next year can be seen. These figures demonstrate some complications in the analysis. For instance the company Harley (on the top) is located at the local maximum, leading to an undefined direction. Mitsubishi and Kanto have similar ratios, which define a "mini-cluster". However, they move away from their centers in almost complete orthogonal directions. Overall we observe a fall in OPM and ATO from 2008 to 2009. This leads to a significant change in the probability density heat map (see figure 8) and motivates a large shift of density center and industrial average.

The quantitative analysis gives statistical values of directional agreement summarized in table 1. The results show that the strongest positive directional agreement is towards the density center on average; followed by the industry average and maximum neighbor target. The median indicates an even stronger directional agreement towards the density center. The median of the directionality of the steepest local ascent is higher than the industry average. Furthermore large variations of the ratios are observed. This suggest the need to consider the whole probability distribution.

The same analysis - as done for the year 2008 - is applied to the years 1995 until 2010. Figure 8 shows the ratios (OPM,ATO) and the corresponding estimated probability density heat maps. The displayed ATO and OPM ranges are [-1.7,8] and [-0.1,0.2] respectively. Most of the years show a single density center. Nevertheless these visualizations indicate that a multivariate parametric normal probability density estimate would be inappropriate. This applies in particular to the years 2007 and 2008 where multiple clusters exist. The quantitative analysis is achieved by aggregating positive and negative directional agreements, which lead to the descriptive statistics shown in table 2. We observe that the number and sum of directional agreements for all targets is greater than the disagreements. The density center is the best target according to all descriptive statistic elements. 
Figure 8: Kernel density heat maps 1995-2010.

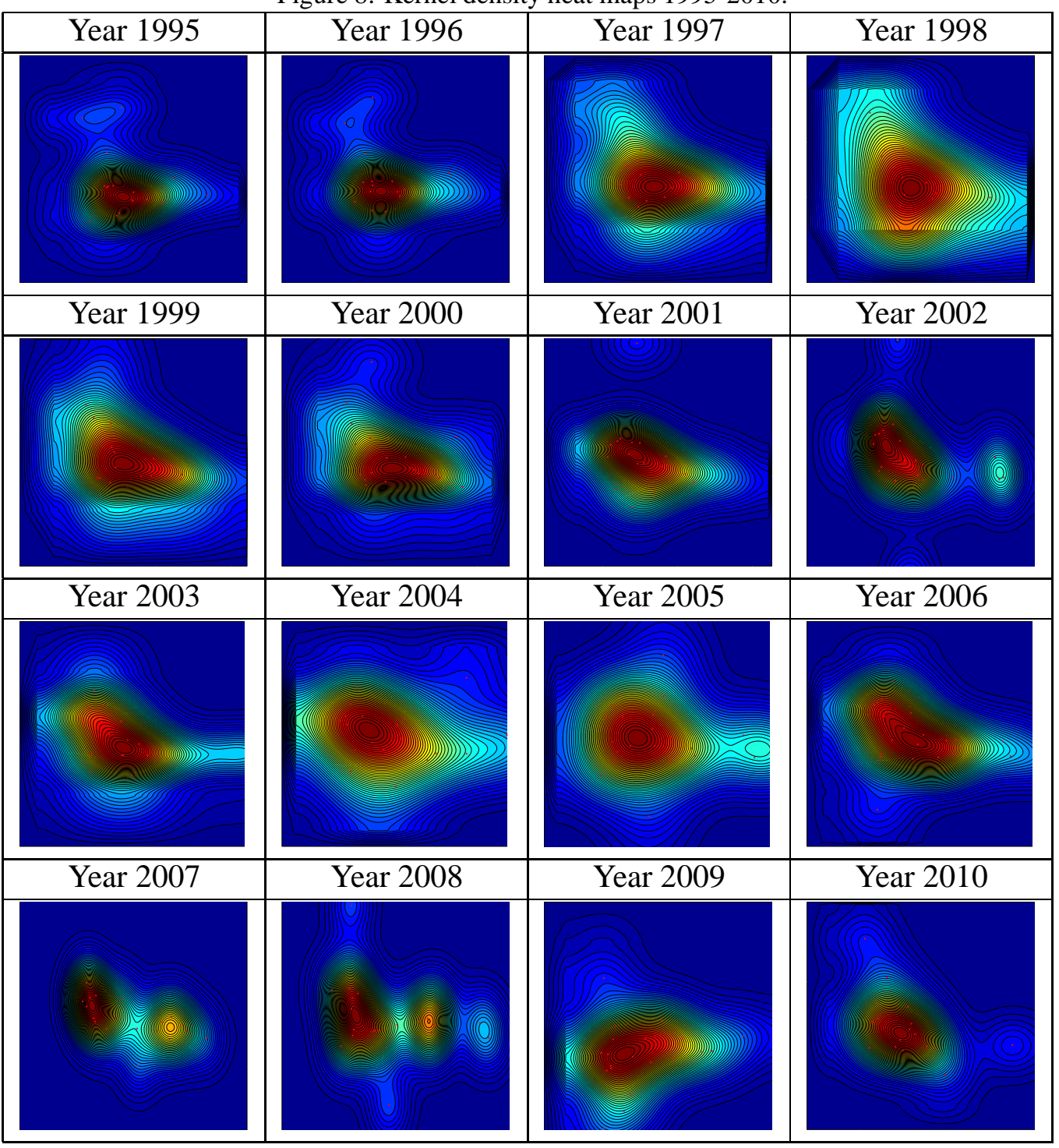


Table 2: Directional agreement, three targets vs actual movement 1995-2009.

\begin{tabular}{rr|rrr}
\hline Directional & Statistic & density & neighbor & ind. avg. \\
\hline Agreement & Mean & 0.544 & 0.486 & 0.517 \\
& Std & 0.280 & 0.280 & 0.290 \\
& Median & 0.578 & 0.498 & 0.554 \\
& Count & 164 & 161 & 150 \\
& Sum & 89.3 & 78.2 & 77.5 \\
\hline Disagreement & Mean & -0.558 & -0.520 & -0.544 \\
& Std & 0.300 & 0.280 & 0.300 \\
& Median & -0.624 & -0.522 & -0.633 \\
& Count & 123 & 126 & 137 \\
& Sum & -68.6 & -65.5 & -74.5 \\
\hline
\end{tabular}

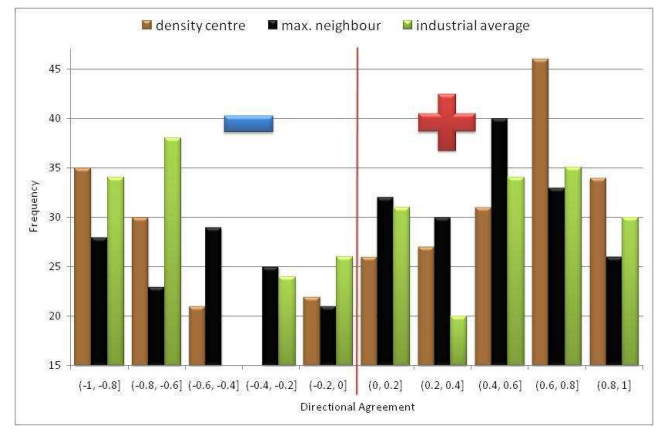

(a)

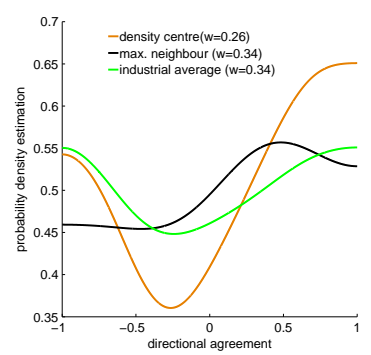

(b)

Figure 9: Directional Agreement distribution (a) Histogram; (b) Probability KDEs.

The positive and negative medians for density center and industry are more pronounced than the neighbor target. A closer examination of the histogram and the density estimation (figure 9) confirms these formations. The probability density estimate for the density center target suggests two distinct groups. One group that is likely to have its DuPont drivers directed towards the density center and the other one going towards the opposite direction. Overall there are $57.1 \%$ positive DuPont drivers over a 16 years period. In table 3 the financial ratios for each financial target from 1995 until 2009 are given by using the count of directional agreements and disagreements.

A refined positive directional agreement overview is obtained by: $\frac{\Psi^{+}}{\Psi^{+}-\Psi^{-}}$where $\Psi^{+}:=\sum[\check{\Psi}>$ $0] \breve{\Psi}$ and $\Psi^{-}:=\sum[\check{\Psi}<0] \check{\Psi}$. Here $\stackrel{\Psi}{ }$ represents the directional agreement of a company $c$ in a specified year $t$ towards a target $\{a, d, m\}$ (e.g. $\stackrel{\Psi}{\Psi}:=\Psi_{c t}^{d}$ ). Using this formula we obtain $\Psi_{a}^{+}=$ $51.0 \%, \Psi_{d}^{+}=56.5 \%$ and $\Psi_{m}^{+}=54.4 \%$. Here the difference between density center and industry 
Table 3: Directional Agreement of DuPont drivers between 1995 and 2009.

\begin{tabular}{r|rrr} 
& density & neighbor & ind. avg. \\
\hline Agreement & 164 & 161 & 150 \\
& $57.1 \%$ & $56.1 \%$ & $52.3 \%$ \\
\hline Disagreement & 123 & 126 & 137 \\
& $42.9 \%$ & $43.9 \%$ & $47.7 \%$ \\
\hline
\end{tabular}

average is $5.5 \%$.

Additionally we analyzed the directional agreement using as the actual direction a 3 years and 5 years future scope. When a 3 years scope is used for determining the actual direction then inclinations to the targets reduce. A further reduction is observed using 5 years for the actual direction, which causes one target to have directional disagreement.

\section{Discussion}

In this paper we studied the profitability of 21 global car manufacturers over a 16-year period in relation to industry-wide profitability targets. To examine whether each firm moved towards targets, we have developed new targets using KDE density estimation, and a new concepts to assess whether there is directional "agreement" between the actual move and the targeted move.

The analysis has shown that firms have a positive directional agreement towards targets: density center, industry average and maximum neighbor. Density center has a $4.8 \%$ better positive directional agreement than the industry average. Furthermore the probability density estimate for the density center allows a better separation between DuPont drivers with directional agreement and disagreement. The profitability probability distributions can be used by companies to adjust their DuPont drivers. Moreover, the probability density estimates (based on a linear diffusion Kernel) capture the yearly dynamics and visualize the overall profitability of a market segment. This can be used by investors to identify profitable company clusters.

The study gives rise to a number of avenues for further research. Following is a discussion of three possible avenues.

The existence of multiple industry averages is consistent with the theory of strategic groups, i.e., groups of firms that tend to gravitate around similar structures, strategies, and performance. 
A brief excursion in the strategic management literature shows a considerable amount of work on the formation and development of strategic groups. For example, Dranove et al. (1998) theorize how strategic interactions within the group influence profitability measures.Nair and Filer (2003) provide corroborating empirical evidence for the existence of groups. Porter (1980) discusses mobility barriers as the primary reason for the formation of groups. It is worth examining clusters of firms in more detail. The advantage of kernel density estimation is that groups remain fluid and confined to the nearest neighbor. Further study may seek to synchronize the results from kernel density estimation with, say, a cluster analysis which explicitly sorts the firms into different clusters.

The second avenue is to further study the impact of different approaches to standardization of the measures and validation of the data points. Scaling the data appropriately is relatively challenging task, and our assumption that the two ratios are of equal importance is relatively crude. We suggest to focus future research on this aspect. Probabilistic methods may be an interesting way forward. We have considered several alternative ways to scale the ratios. Using only the maximum gives emphasis to positive ratios. Another approach was assuming normal distributed data and scaling by using the mean plus two or three standard deviations. We considered the effects of two more ways: not scaling the data and using the preprocessed ranges. Not to scale the data is materially similar to not using the OPM ratios, so this is not an option. Lastly we used the data preprocessing ranges, which dampens the data slightly more. However, this has the advantage that the scaling is independent of the year. Scaling on yearly basis might be problematic for comparison purposes. Further investigation is recommended.

A third possible avenue is to look at generalizations of movements, for instance the dynamics of movements. We have looked at strategic movements in two-dimensional space. One of the interesting aspects of further research is to look at multi-dimensional strategic directions. One dimension could be related to working capital management (i.e., trying to remain liquid while attempting to become more profitable). This would lead to strategic movements in three dimensions. Kernel density estimations can be easily extended to higher dimensions, and a contour map could be projected on a unit sphere to examine the dynamics within an industry. The above opens up the 
statistical calculus of directions to profitability analysis.

Much of the dynamics of an industry can be described by the degree to which the firms react to each other. This is particularly so with respect to levels of profitability. Individual and group dynamics of companies are therefore an interesting area of further investigation. The conceptual apparatus developed in this paper opens up the way for such analysis.

\section{Appendix}

The linear diffusion KDE can be best understood by characterizing the Gaussian kernel density estimator:

$$
f(x, w)=\frac{1}{n} \sum_{k=1}^{n} \phi\left(x-y_{k}, w\right), \quad x \in \mathbb{R},
$$

where $w$ is the bandwidth, $\left\{y_{1}, \ldots, y_{n}\right\}$ are independent realizations and

$$
\phi\left(x-y_{k}, w\right)=\frac{1}{w \sqrt{2 \pi}} e^{-\frac{1}{2}\left(\frac{x-y_{k}}{w}\right)^{2}} .
$$

This estimator has the characteristic that it is the unique solution of the diffusion partial differential equation (PDE):

$$
\frac{\partial}{\partial w} f(x, w)=\frac{1}{2} \frac{\partial^{2}}{\partial w^{2}} f(x, w), \quad x \in \mathbb{R}, w>0,
$$

and the initial condition $f(x, 0)=\frac{1}{n} \sum_{k=1}^{n}\left[x=y_{k}\right]$. This motivates the following generalization:

$$
\frac{\partial}{\partial w} g(x, w)=\operatorname{Lg}(x, w), \quad x \in \mathbb{R}, w>0
$$

where the linear differential operator is defined by the form $\frac{1}{2} \frac{d}{d x}\left(a(x) \frac{d}{d x}\left(\frac{\dot{p}}{p(x)}\right)\right)$. Here $a$ and $p$ are positive real functions with bounded second derivatives. The initial condition for $g(0, w)$ is $\frac{1}{n} \sum_{k=1}^{n}\left[x=y_{k}\right]$ and the boundary condition is $\frac{\partial}{\partial x} \frac{g(x, t)}{p(x)}=0$. Note if $a=1$ and $p=1$ we will obtain equation 15 . The solution of the linear diffusion partial differential equation:

$$
g(x, w)=\frac{1}{n} \sum_{k=1}^{n} \kappa\left(x, y_{k}, t\right)
$$


will be called linear diffusion estimator and $\kappa$ diffusion kernel.

Further details on this kind of kernel density estimation can be found in Botev et al. (2010).

Botev, Z. I., Grotowski, J. F., Kroese, D. P., 2010. Kernel density estimation via diffusion. Annals of Statistics.

Bowman, A. W., Azzalini, A., 1997. Applied Smoothing Techniques for Data Analysis. Oxford University Press, Oxford.

Dranove, D., Peteraf, M., Shanley, M., 1998. Do strategic groups exist? an economic framework for analysis. Strategic Management Journal 19 (11), 1029-1044.

Fairfield, P. M., Yohn, T. L., 2001. Using asset turnover and profit margin to forecast changes in profitability. Review of Accounting Studies 6, 371-385.

Fama, E. F., French, K. R., 2000. Forecasting profitability and earnings. Journal of Business 73 (2), $161-175$.

Freeman, R. N., Ohlson, J. A., Penman, S. H., 1982. Book rate-of-return and prediction of earnings changes: an empirical investigation. Journal of Accounting Research 20 (2), 639-653.

Hastie, T., Tibshirani, R., Friedman, J., February 2009. The Elements of Statistical Learning, Second Edition: Data Mining, Inference, and Prediction, 2nd Edition. Springer Series in Statistics. Springer.

Lev, B., 1969. Industry averages as targets for financial ratios. Journal of Accounting Research 7 (2), 290-299.

Nair, A., Filer, L., 2003. Co-integration of firm strategies within groups: a long-run analysis of firm behavior in the Japanese steel industry. Strategic Management Journal 24 (2), 145-159.

Penman, S., 2010. Financial statement analysis and security valuation, 4th Edition. McGraw-Hill, New York.

Porter, M. E., 1980. Competitive strategy. The Free Press, New York. 
Porter, M. E., 1985. Competitive advantage: creating and sustaining superior performance. The Free Press, New York.

Saranga, H., 2009. The Indian auto component industry: estimation of operational efficiency and its determinants using DEA. European Journal of Operational Research 196, 707-718.

Scott, D. W., Sep. 1992. Multivariate Density Estimation: Theory, Practice, and Visualization (Wiley Series in Probability and Statistics). Wiley.

Silverman, B., 1986. Density Estimation for Statistics and Data Analysis. Chapman and Hall.

So, M. M., Thomas, L. C., 2011. Modelling the profitability of credit cards by Markov decision processes. European Journal of Operational Research 212, 123-130.

Soliman, M. T., 2004. Using Industry-Adjusted DuPont Analysis to Predict Future Profitability. SSRN eLibrary.

Soliman, M. T., 2008. The use of DuPont analysis by market participants. The Accounting Review $83(3), 823-853$.

Tecles, P. L., Tabak, B. M., 2010. Determinants of bank efficiency: the case of Brazil. European Journal of Operational Research 207, 1587-1598.

Wu, C.-R., Lin, C.-T., Tsai, P.-H., 2010. Evaluating business performance of wealth management banks. European Journal of Operational Research 207 (971-979). 